\title{
Transition to adulthood: A critical role for exercise in building overall well-being
}

\author{
Channakeshava Sokke Umeshappa ${ }^{1^{*}}$, Ryan Punambolam ${ }^{2}$ \\ ${ }^{1}$ Department of Microbiology, Immunology and Infectious Diseases, Institute of Inflammation, Infection and Immunity, Faculty of \\ Medicine, University of Calgary, Calgary, Canada; ${ }^{*}$ Corresponding Author: chs221@mail.usask.ca \\ ${ }^{2}$ Division of neurology, Department of Medicine, Fraser Health Authority, Vancouver, Canada
}

Received 2 May 2013; revised 3 June 2013; accepted 28 June 2013

Copyright (c) 2013 Channakeshava Sokke Umeshappa, Ryan Punambolam. This is an open access article distributed under the Creative Commons Attribution License, which permits unrestricted use, distribution, and reproduction in any medium, provided the original work is properly cited.

\section{ABSTRACT}

A growing number of multidisciplinary studies have reported the beneficial effects of physical activities towards the development of overall well-being and the reduction of chronic illnesses. Physical inactivity is a serious worldwide problem and is considered the key lifestyle factor that negatively influences health and function of individuals. Here we propose that growth kinetics of body and mind and associated disproportionate physical activity may have considerable effects on the increased physical and mental illnesses and premature death rates currently observed in mid to late adulthood people. Children and young adults, being in exponential growth phase and self-motivated to engage in physical activity, possess more cognition, resilience, and mind-body-emotion-spirit integration. In contrast, as our body transitions into adulthood, there is a decline in the growth of body and mind. Furthermore, in this period, adults experience the increased stressors of career and financial demands, time pressure, and family responsibilities. The decline in growth combined with prolonged stress appears to result in reduced cognition, resilience, and mind-bodyemotion-spirit integration, leading to sedentary and other faulty lifestyles, which could further lead to a predisposition to maladaptive behaviours and various chronic diseases at a later stage. In agreement with this, currently more than two thirds of the adult population across the world are physically inactive and are failing to perform the daily recommended guidelines of moderate to vigorous exercise. As negative changes and faulty lifestyles begin to occur during and after adulthood transition, creating awareness in people between 29 to 35 years of age about mindful exercise should be considered a national priority. This would allow adults to achieve overall well-being, and increased productivity and life expectancy, reducing health care-associated economic burden placed on the nation.

Keywords: Physical Activity; Adult Transition Phase; S-Shaped Human Mind and Body Sigmoid Curve; Well-Being; Chronic Physical and Mental Illnesses

\section{INTRODUCTION}

A vast majority of physical illnesses (e.g. cardiovascular diseases, diabetes, cancer, and obesity) and mental illnesses (e.g. mood disorders, anxiety disorders, personality disorders, eating disorders, schizophrenia, and addictions) develop at an increasing rate with age, especially in the later years of life (30 - 59 years) $[1,2]$. These illnesses are inversely correlated to our overall well-being, which is in turn linked to emotional, physical, mental and spiritual health, and are the main causes of increased health care expenditures worldwide. Elucidating what makes these components work together to reach overall well-being is therefore necessary to transform our public physical and mental health.

Currently, researchers are in search of defining causes that predispose the adults to physical and mental illnesses at later stages in life. Among many lifestyle factors identified, physical exercise is considered to be one of the key lifestyle factors that determine overall wellbeing of the individuals [3,4]. Previously, studies in children and young adults have shown that exercise decreases physical disorders and improves mental health 
and academic performance by increasing brain health and functions [5-7]. Similarly, exercise enhanced cognition in older adulthood considerably, although it is shown to be less effective in young adults $[8,9]$. When and how exercise influences well-being in adulthood that require further research. In this report, we propose that adulthood transition (29 - 35 yr of age) is the defining stage that, if attention to health not given, involves several negative changes in the life styles, predisposing the adult to various physical and mental illnesses in the later stages. Early intervention during and after adulthood transition through exercise are critical in developing and maintaining overall well-being, thereby in increasing the lifespan of the individual.

\section{LIFE AS A "SIGMOID CURVE"}

In evolutionary terms, as with all higher animals, our life cycle (both mental and physical) follows a characteristic "S-shaped sigmoid curve" pattern (Figure 1) [9-11]. It is comprised of 1) lag period (infantile and early childhood, birth to 5 yr of age) where body and mind show minimal growth and start preparing for growth; 2) logarithmic/exponential phase (Juvenile and young adulthood, 6 to 29 yr of age) where body and mind growth begins slowly in the beginning and become rapid subsequently; 3) stationery/deccelerating phase (adulthood, 30 to 59 yr of age) where growth continues slowly and finally stops completely; and 4) decline phase (older adulthood, 59 to 100 yr of age), growth of body and mind ceases completely and prepares for death. (Figure 1). Both growth kinetics of our body and mind, and associated corresponding life styles appear to play an important role in achieving our resilience (an individual's ability to recover following a negative life event) [12], and mind-body-emotion-spirit integration. Perhaps due to the growth phase and increased physically active lifestyle, children and young adults often exhibit enhanced cognition, resilience and mind-body-emotionspirit integration, thereby boosting their physical and mental health [8]. However, as our body transitions into adulthood, we gradually change lifestyles and begin to neglect body, mind, emotions and spirit due to prolonged stressors of career and financial demands, time pressure and family responsibilities. These stressors considerably retard psychomotor functions in adulthood, further precipitating the loss of mind-body-emotion-spirit integra-

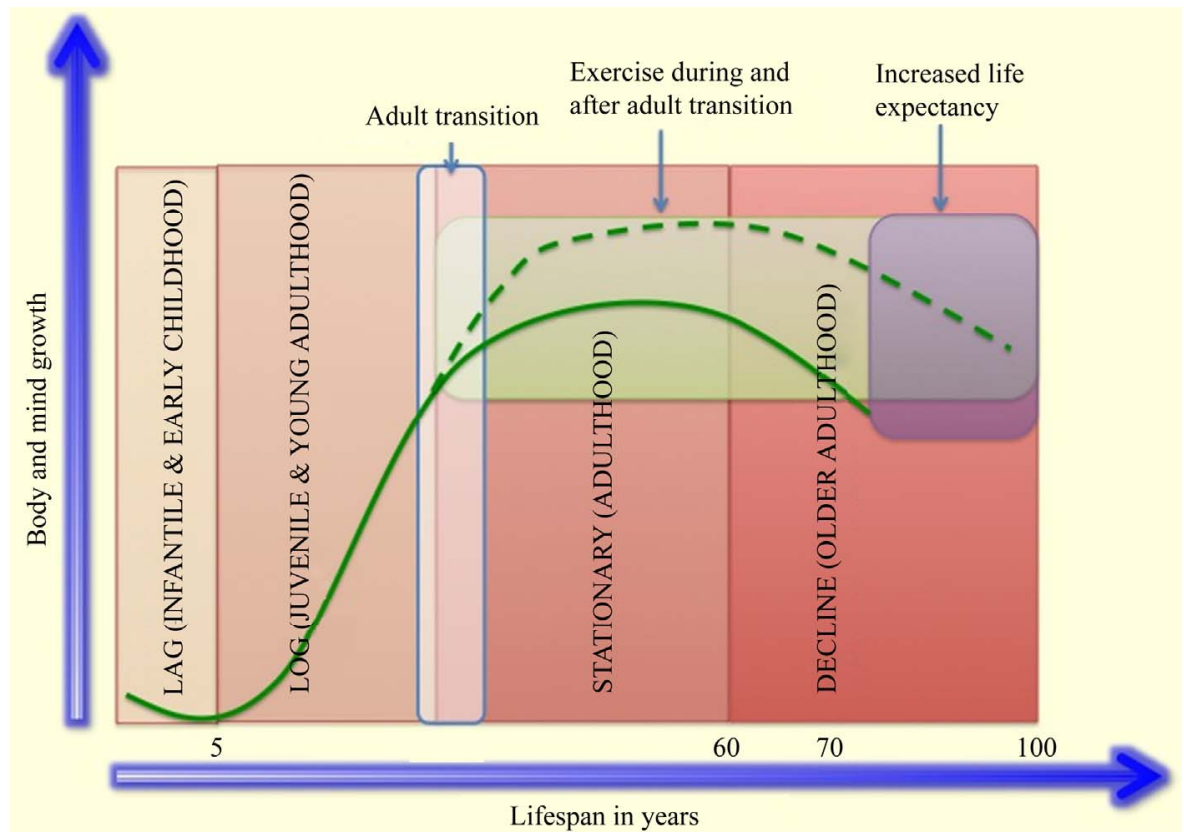

Figure 1. Characteristics S-Shaped sigmoid human body and mind growth curve. Lag period (infantile and early childhood, birth to 5 years of age), is the first phase during growth where body and mind show minimal growth and prepares for growth. In logarithmic/exponential phase (Juvenile and young adulthood, 6 to 29 years of age), growth begins slowly in the beginning and becomes rapid subsequently. In stationery/decelerating phase (adulthood, 30 to 59 years of age), growth continues slowly and finally stops completely. In decline phase (older adulthood, 59 to 100 years of age), growth of body and mind cease completely and prepare for death. The productivity, overall well-being (not shown) and life expectancy of human can be increased by proper intervention through physical exercise and meditation, particularly during and after adult transition phase (29 to 35 years of age) where sufficient reserves and energy in body are still available. 
tion. In contrast to children and young adults, physical activity and cognitive functions of adults are considerably different $[9,13]$. For instance, while adults tend to lead sedentary lifestyles, children are much more active physically and mentally. If we observe carefully, in any given awake time, children exhibit more movements (e.g. hands and legs)/minute, often perform many of the yoga stances, and exhibit increased laughing rates. In contrast, adults tend to show reduced body movements, perform no yoga stances, and exhibit reduced laughing rates. The loss of mind-body-emotion-spirit integration may also cause the adults to experience a high degree of the six passions of mind enemies (lust, greed, anger, delusory emotional attachment, jealousy, and pride), and make them vulnerable to gangs, crimes, drugs and violence [14]. One or several of these factors in turn contribute to faulty life styles, including physical inactivity, and irregular sleep and food patterns, as seen in majority of the adults worldwide [15]. If this cycle continues from adult transition into adulthood, the individual will be inclined to develop negative personality traits and eventually become susceptible to various chronic diseases (both physical and mental disorders). For instance, many doctoral and postdoctoral trainees, who were extremely successful in their earlier life, suddenly experience academic failure for the first time, face difficulty in dealing with supervisors, or develop physical and mental illnesses when they reach adulthood [13]. This situation in adults appears to be even worse in under developed and developing countries, possibly due to poor literacy. Lack of physical exercise and assuming other maladaptive coping behaviours such as smoking, poor adherence to medical regimens, increased social isolation, excess alcohol consumption and illicit drugs use are other important pathway by which chronic stressors influence disease risk in early to mid-adulthood [14]. Thus, physical inactivity and increased stressors associated with adulthood transition appear to be one of the key determining factors for poor productivity, and increased vulnerability of adults for chronic diseases and/or maladaptive coping behaviours.

Attaining consciousness to our health is the greatest challenge in life. Although all adults experience the above effects, only those who pay attention to health and acquire resilience become successful in their life. Those who achieve this consciousness rejuvenate and learn to balance emotional, physical, mental and spiritual health. Exercise becomes an important component of their life styles. Unfortunately, a vast majority of the global population is living a life of poor consciousness and negatively impacting a nation's wealth, health and peace. Interestingly, a large proportion of this category seems to have neglected their health during adult transition period where much attention to health is required. If this is true, there is an urgent need to intervene the adult transition phase while much reserves and energy within our body are still available to increase our productivity, life expectancy, and resistance against physical and mental illnesses (Figure 1) [10,16].

\section{A DIET OF EXERCISE TO BUILD PHYSICAL AND MENTAL HEALTH}

Fortunately, the loss of body and mind consciousness, which leads to poor productivity and predisposition to physical and mental illnesses, is not a normal part of the aging process in adulthood. Hence, the adverse effects that occur in adulthood can be readily prevented through proper intervention methods once an individual reaches adulthood. A growing number of multidisciplinary studies have reported the beneficial effects of physical activities such as cardiovascular exercise, yoga, and meditation, during adulthood. Importantly, these activities may improve physical, emotional and spiritual resilience while decreasing the incidence of physical and mental disorders and increasing the lifespan of the individual (Figure 1) [5,17]. Practice guidelines emphasize the role of cardiovascular exercise as a comprehensive treatment for depression and anxiety disorders $[18,19]$. Physical activity is shown to be critical for improving stress management and supporting cessation of maladaptive behaviours, such as smoking [20,21]. Indeed, physical inactivity is considered as a major risk factor for heart disease and stroke, high blood pressure, high blood cholesterol, cancers, diabetes, and obesity [3,22]. Mechanistically, cardiovascular exercises, such as aerobics, may improve resilience, thereby reducing the incidence of mood disorders by increasing plasma monoamines, attenuation of the hypothalamic-pituitary-adrenal (HPA) axis in response to acute or chronic stressors, release of beta-endorphins [5,23]. In addition, cardiovascular exercise may be associated with increases in cerebral blood volume, neurochemicals such as brain-derived neurotrophic factors, brain neovasculature, and neurogenesis in hypothalamus, which in turn influence anterior cingulate cortex that process sensory, motor, emotional and cognitive information $[5,24,25]$. Similarly, meditation, which emphasizes present moment non-judgemental awareness, is associated with modifications of HPA axis, improved cerebral circulation, neurogenesis and secretion of neuroprotectives, such as GABA, reduction of amygdala volume and increased communication with different organ systems $[17,26,27]$. Over thousands of years, yoga and meditation have been developed to build mental health, and to increase our self-consciousness. They demand deep breathing, concentration and calmness, and actively engage the brain and nerve centers situated all along the musculoskeletal systems thereby help in improving brain health $[28,29]$. As a proof of concept, it has been estimated that $33 \%$ of all deaths related to coronary heart disease, $20 \%$ of deaths related to type 2 
diabetes, $20 \%$ of deaths related to hypertension, and $25 \%$ of deaths related to stroke can be avoided if all Canadians perform 60 minutes of physical exercise per day [22]. Hence, mindful exercises can be used as proper intervention method during and after adulthood transition to increase overall well-being of the individual.

\section{FUTURE FOCUS FOR THE CURRENT REALITY}

Recent data further suggests that adults are growing increasingly sedentary and weak, and that these lifestyle aspects are associated with an earlier onset of several chronic diseases (both physical and mental) [30,31]. Adults working in universities and job centers have minimal access to fitness centers, at least in the majority of underdeveloped and developing countries. Even in developed countries, although fitness centers exist, only a small percent of adults are motivated to engage in exercise. For example, in the USA, 74\% of adults fail to follow the recommended guideline of a minimum of 30 minutes of moderate exercise per day [5]. Strikingly, this proportion is even higher in Canada where currently 85\% of adults do not meet the recommended guideline of 150 minutes of moderate to brisk aerobic exercise per week, accrued in sessions of $10 \mathrm{~min}$ or higher [29,32]. These percentages appear to be even higher in underdeveloped and developing countries, possibly due to low literacy. Accordingly, about two-thirds of North Americans, and one billion people globally are estimated to be overweight or clinically obese due to physical inactivity, and this is one of the root causes behind many chronic physical and mental illnesses [33]. Thus, healthcare cost arising from sedentary lifestyles, principally in adulthood and older adulthood, is considered to be high in both developing and developed countries. For example, physical inactivity alone accounted US $\$ 76$ billion in medical costs in the year 2000 [5,34]. Similarly, it was alone responsible for $\$ 5.3$ billion in direct and indirect costs in 2001 [30]. Apart from healthcare costs, decreased wellbeing of adults also results in poor working environment and decreased productivity of the adults.

It has been reported that exercise is relatively more critical for adults than for children and younger adults to maintain physical and mental health [9]. As adults are the backbone of any country and their productivity in working area is directly related to country's economy, creating awareness about the importance of mindful exercise in achieving overall well-being should be considered a national priority. As decline of resilience, and mind-body-emotion-spirit integration, and increased stress during adulthood transition subsequently leads to an increases in associated heart diseases, diabetes, high blood pressure, cancers, depression and anxiety, obesity, development of negative personality, and a decrease in lifespan (Figure 1), a particular emphasis should be given to people in the age range, 29 to $35 \mathrm{yr}$ (e.g. doctoral and postdoctoral trainees, and young scientists) to create awareness about exercise through regular workshops and seminars. Physical activity and sports should be promoted for adults in all the job centers and universities across the world. More epidemiological and molecular studies should be carried out to determine the factors in adulthood that influence changes leading to faulty life styles, decreased resilience and mind-bodyemotion-spirit integration, and increased predisposition to physical and mental illnesses, and how physical exercise and meditation help alleviate these negative effects. As body and mind growth rates vary considerably during the human life cycle, studies should also be focused on determining both qualitative and quantitative physical exercise program, tailoring to the needs of each age category. It has been estimated that almost $85 \%$ of all the current diseases are derived from imbalanced lifestyles [35]. Policy makers at federal, provincial/territorial and municipal governments, community planners and developers, and school boards should make sure mindful exercise and meditation becomes an integral part of the adult lifestyle to achieve overall well-being and to reduce economic burden placed on the nation. Prevention is always better than cure.

\section{ACKNOWLEDGEMENTS}

Authors sincerely thank Johanna Zaal DeLongchamp, research technician, University of Calgary, Calgary, Alberta, and Dr. Sateesh Kaagale, Assistant Research Officer at National Research Council, Saskatoon, Saskatchewan for feedback on the article. Dr. Umeshappa is supported by the postdoctoral fellowship from CIHR and Canadian Association for the Study of the Liver. Dr. Punambolam is neurologist in Division of Neurology, Fraser Health Authority, BC, Canada.

\section{REFERENCES}

[1] American Medical Network, Inc. (2011) Major depressive disorder. http://www.health.am/psy/major-depressive-disorder

[2] Grant, B.F., Hasin, D.S., Stinson, F.S., Dawson, D.A., June Ruan, W., Goldstein, R.B., Smith, S.M., Saha, T.D. and Huang, B. (2005) Prevalence, correlates, co-morbidity, and comparative disability of DSM-IV generalized anxiety disorder in the USA: Results from the national epidemiologic survey on alcohol and related conditions. Psychological Medicine, 35, 1747-1759. doi:10.1017/S0033291705006069

[3] Heart and Stroke Foundation of Canada Position Statement (2011) Physical activity, heart disease and stroke. http://www.heartandstroke.com/site/c.ikIQLcMWJtE/b.5 $\underline{263145}$

[4] (2000) Promoting better health for young people through physical activity and sports: A report to the president 
from the secretary of health and human services and the secretary of education. http://www2.ed.gov/offices/OSDFS/physedapndc.pdf

[5] Hillman, C.H., Erickson, K.I. and Kramer, A.F. (2008) Be smart, exercise your heart: Exercise effects on brain and cognition. Nature Reviews Neuroscience, 9 58-65. doi:10.1038/nrn2298

[6] Sibley, B.A. and Etnier, J.L. (2003) The relationship between physical activity and cognition in children: A metaanalysis. Pediatric Exercise Science, 15, 243-256.

[7] Pierson, W.R. and Montoye, H.J. (1958) Movement time, reaction time, and age. The Journals of Gerontology, 13, 418-421. doi:10.1093/geronj/13.4.418

[8] Salthouse, T.A. and Davis, H.P. (2006) Organization of cognitive abilities and neuropsychological variables across the lifespan. Developmental Review, 26, 31-54. doi:10.1016/j.dr.2005.09.001

[9] Spirduso, W.W. (1980) Physical fitness, aging, and psychomotor speed: A review. The Journals of Gerontology, 35, 850-865. doi:10.1093/geronj/35.6.850

[10] Rittler, G. (2008) Born. Grow. Mature. Decline. Die? The why of change.

http://claritynew.kanonclarity.com/?p=564

[11] (2010) Growth, regeneration and ageing: Types of growth. http://www.tutorvista.com/content/biology/biology-iv/gro wth-regeneration-ageing/growth-types.php

[12] Southwick, S.M., Vythilingam, M. and Charney, D.S. (2005) The psychobiology of depression and resilience to stress: Implications for prevention and treatment. Annual Review of Clinical Psychology, 1, 255-291. doi:10.1146/annurev.clinpsy.1.102803.143948

[13] Gewin, V. (2012) Mental health: Under a cloud. Nature 490, 299-301. doi:10.1038/nj7419-299a

[14] (2000) Promoting better health for young people through physical activity and sports: A report to the president from the secretary of health and human services and the secretary of education. http://www2.ed.gov/offices/OSDFS/physedapndc.pdf

[15] Thirlaway, K. and Upton, D. (2009) The psychology of lifestyle: Promoting health behaviour.

http://xa.yimg.com/kq/groups/20750879/611665519/nam e/Psychology+of+Lifestyle.pdf

[16] Mark, P. (2011) The SBC and the near future of FBCTC. http://www.fbcthecolony.org/rwt-blog-24035

[17] Streeter, C.C., Whitfield, T.H., Owen, L., Rein, T., Karri, S.K., Yakhkind, A., Perlmutter, R., Prescot, A., Renshaw, P.F., Ciraulo, D.A. and Jensen, J.E. (2010) Effects of yoga versus walking on mood, anxiety, and brain GABA levels: A randomized controlled MRS study. Journal of Alternative and Complementary Medicine, 16, 1145-1152. doi:10.1089/acm.2010.0007

[18] Shephard, R.J. (1997) Exercise and relaxation in health promotion. Sports Medicine, 23, 211-217. doi:10.2165/00007256-199723040-00001

[19] Barbour, K.A. and Blumenthal, J.A. (2005) Exercise training and depression in older adults. Neurobiology of Aging, 26, 119-123. doi:10.1016/j.neurobiolaging.2005.09.007

[20] deRuiter, W. and Faulkner, G. (2006) Tobacco harm re- duction strategies: The case for physical activity. Nicotine \& Tobacco Research, 8, 157-168. doi:10.1080/14622200500494823

[21] Marcus, B.H., Lewis, B.A., King, T.K., Albrecht, A.E., Hogan, J., Bock, B., Parisi, A.F. and Abrams, D.B. (2003) Rationale, design, and baseline data for commit to quit II: An evaluation of the efficacy of moderate-intensity physical activity as an aid to smoking cessation in women. Preventive Medicine, 36, 479-492. doi:10.1016/S0091-7435(02)00051-8

[22] Warburton, D.E.R., Katzmarzyk, P.T., Rhodes, R.E. and Shephard, R.J. (2007) Evidence-informed physical activity guidelines for Canadian adults. Applied Physiology, Nutrition and Metabolism, 32, S16-S68. doi:10.1139/H07-123

[23] Koseoglu, E., Akboyraz, A., Soyuer, A. and Ersoy, A.O. (2003) Aerobic exercise and plasma beta endorphin levels in patients with migrainous headache without aura. $\mathrm{Ce}$ phalalgia, 23, 972-976. doi:10.1046/j.1468-2982.2003.00624.X

[24] Young, S.N. (2007) How to increase serotonin in the human brain without drugs. Journal of Psychiatry and Neuroscience, 32, 394-399.

[25] van Praag, H., Kempermann, G. and Gage, F.H. (1999) Running increases cell proliferation and neurogenesis in the adult mouse dentate gyrus. Nature Neuroscience, 2, 266-270. doi:10.1038/6368

[26] MacLean, C.R., Walton, K.G., Wenneberg, S.R., Levitsky, D.K., Mandarino, J.P., Waziri, R., Hillis, S.L. and Schneider, R.H. (1997) Effects of the transcendental meditation program on adaptive mechanisms: Changes in hormone levels and responses to stress after 4 months of practice. Psychoneuroendocrinology, 22, 277-295. doi:10.1016/S0306-4530(97)00003-6

[27] McEvoy, M. (2012) Exploring the stress response: HPA axis, nutrition, emotions \& the limbic system. http://metabolichealing.com/key-integrated-functions-ofyour-body/hormone-and-endocrine/exploring-the-stress-r esponse-hpa-axis-nutrition-emotions-and-the-limbic-syste $\underline{m}$

[28] Cotman, C.W., Berchtold, N.C. and Christie, L.A. (2007) Exercise builds brain health: Key roles of growth factor cascades and inflammation. Trends in Neurosciences, 30, 464-472. doi:10.1016/j.tins.2007.06.011

[29] Vaynman, S. and Gomez-Pinilla, F. (2006) Revenge of the "sit": How lifestyle impacts neuronal and cognitive health through molecular systems that interface energy metabolism with neuronal plasticity. Journal of Neuroscience Research, 84, 699-715. doi:10.1002/jnr.20979

[30] Katzmarzyk, P.T., Gledhill, N. and Shephard, R.J. (2000) The economic burden of physical inactivity in Canada. CMAJ, 163, 1435-1440.

[31] Katzmarzyk, P.T., Gledhill, N. and Shephard, R.J. (2000) The economic burden of physical inactivity in Canada. CMAJ, 163, 1435-1440.

[32] Colley, R.C., Garriguet, D., Janssen, I., Craig, C.L., Clarke, J. and Tremblay, M.S. (2011) Physical activity of Canadian adults: Accelerometer results from the 2007 to 2009 Canadian health measures survey statistics Canada. 
http://www.statcan.gc.ca/pub/82-003-x/2011001/article/1 1396-eng.htm

[33] Pearson, H. (2006) Medicine: Sleep it off. Nature, 443, 261-263. doi:10.1038/443261a

[34] Pratt, M., Macera, C.A. and Wang, G. (2000) Higher direct medical costs associated with physical inactivity.
Physician and Sportsmedicine, 28, 63-70. doi:10.3810/psm.2000.10.1237

[35] Lyons, M. (1996) The self-healing method-A physiotherapy for the 21st century.

http://www.positivehealth.com/article/physiotherapy/the-self -healing-method-a-physiotherapy-for-the-21st-century) 